\title{
Discordance between TSTs and IFN- $\gamma$ release assays: the role of NTM and the relevance of mycobacterial
}

sensitins

\section{To the Editors:}

We read with interest the recent study by LATORRE et al. [1] entitled "Evaluating the non-tuberculous mycobacteria effect in the tuberculosis infection diagnosis". We agree that discordance between tuberculin skin tests (TSTs) and interferon (IFN)- $\gamma$ release assays (IGRAs) presents physicians with a considerable management dilemma when evaluating children for latent tuberculosis (TB) infection (LTBI) in routine clinical practice. We have previously urged caution in the interpretation of discordant results and have highlighted this area as a research priority [2,3]. We therefore commend the authors for investigating a potential underlying cause of discordance. However, we believe that the interpretation of the data presented by LATORRE et al. [1] is based on erroneous assumptions, and that as a result the conclusions are overstated. We suggest that a more cautious and contextualised interpretation of the study findings is warranted.

As indicated by LATORRE et al. [1], previous bacille CalmetteGuérin immunisation and exposure to non-tuberculous mycobacteria (NTM) are frequently cited as the primary factors underlying discordance between TSTs and IGRAs, although convincing data to support these concepts are currently lacking. In the study by LATORRE et al. [1], children with suspected LTBI were assessed with a TST, a commercial IFN- $\gamma$ ELISpot assay (the T.SPOT.TB assay, incorporating early secretory antigenic target 6 (ESAT-6) and culture filtrate protein 10 (CFP-10)) and an in-house IFN- $\gamma$ ELISpot assay using Mycobacterium avium sensitin (MAS) as the stimulating antigen. In the subgroup of children that had TST+/T.SPOT.TB- discordance, $47.6 \%$ showed a "positive" response in the IFN- $\gamma$ ELISpot using MAS as the stimulant (contrasting with the absence of response to ESAT-6 and CFP-10). The authors interpret this observation as evidence that previous NTM sensitisation in these children resulted in a false-positive TST result and thereby discordance.

While we agree that this is one possible explanation, there is an alternative explanation that would equally account for these observations. Importantly, significant cross-reactivity between different mycobacterial sensitins has been previously consistently shown in animal models [4]. Furthermore, more than a decade ago, LEIN et al. [5] convincingly demonstrated that $\mathrm{T}$ cell assays incorporating MAS cannot reliably distinguish between $M$. tuberculosis and $M$. avium complex (MAC) infection in humans. In that study, the authors used the same MAS preparation as LATORRE et al. [1] to assess T-cell responses in adults with culture-confirmed TB $(n=27)$ or MAC $(n=10)$ infection. Somewhat unexpectedly, higher mean IFN- $\gamma$ concentrations were observed in supernatants from peripheral blood mononuclear cells stimulated with MAS in patients with
TB than those with MAC infection. In addition, MAS-sensitised T cells were detected in the majority of patients with TB. These data strongly suggest that there is considerable cross-reactivity between antigens encountered by the human immune system during M. tuberculosis infection and antigens contained in MAS. We also note that in the study by LATORRE et al. [1], in the subgroup of children that were TST+/T.SPOT.TB+ (and therefore highly likely to have LTBI), 50\% showed a "positive" response to MAS in the in-house IFN- $\gamma$ ELISpot assay, which further questions the ability of MAS-based assays to discriminate between TB and NTM infection, or alternatively exposure.

The limited ability of MAS to distinguish between different mycobacterial infections is not surprising. Unlike the welldefined peptides ESAT-6 and CFP-10, which are thought to be relatively $M$. tuberculosis-specific (despite orthologues of these proteins being present in several other mycobacterial species including M. kansasii, M. marinum and M. szulgai), MAS is a mixture of heterogenous mycobacterial antigens, analogous to the purified protein derivative used in the TST [6]. Crossreactivity with other mycobacterial species is therefore likely to occur, as indicated by the manufacturer's warning mentioned by LATORRE et al. [1], that is particularly likely to be the case with $M$. intracellulare and M. scrofulaceum.

Taken together, these facts make it questionable whether the observations by LATORRE et al. [1] in the subgroup of children with TST+/T.SPOT.TB- discordance truly reflect previous NTM exposure. An alternative explanation is that the assays using MAS detected T-cell sensitisation resulting from previous $M$. tuberculosis exposure and/or LTBI (i.e. confirming the positive TST), while the T.SPOT.TB produced a false-negative result. Published data show that up to one-third of children with culture-proven active $\mathrm{TB}$ have negative or indeterminate T.SPOT.TB assay results [7], which highlights the limitations of these assays and lends support to the latter explanation. In the absence of a gold standard for LTBI, neither hypothesis can be tested with certainty. However, given these uncertainties we believe it is premature of the authors to suggest that chemoprophylaxis could be safely withheld in these patients. Contrary to the authors' assertions, we believe their study does not provide "enough evidence" to justify changes in clinical practice.

We concur with LATORRE et al. [1] that there remains an urgent need to explore the immunology of underlying discordance between TSTs and IGRAs in greater detail. However, in view of the comparatively poor performance of IGRAs in children and the uncertainties surrounding their interpretation we, and other researchers in this field, firmly believe that research to identify better biomarkers and immunological correlates of TB infection remains crucial [3]. 


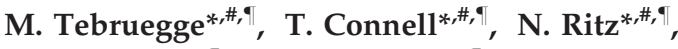

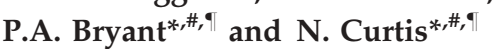

*Dept of Paediatrics, The University of Melbourne, ${ }^{\#}$ Infectious Diseases Unit, Dept of General Medicine, and "Murdoch Children's Research Institute, Royal Children's Hospital Melbourne, Parkville, Victoria, Australia.

Correspondence: M. Tebruegge, Dept of Paediatrics, The University of Melbourne, Royal Children's Hospital Melbourne, Parkville, VIC 3052, Australia. E-mail: marc.tebruegge@rch. org.au

Statement of Interest: None declared.

\section{REFERENCES}

1 Latorre I, De Souza-Galvao M, Ruiz-Manzano J, et al. Evaluating the non-tuberculous mycobacteria effect in the tuberculosis infection diagnosis. Eur Respir J 2010; 35: 338-342.

2 Connell T, Tebruegge M, Ritz N, et al. Interferon-gamma release assays for the diagnosis of tuberculosis. Pediatr Infect Dis J 2009; 28: 758-759.

3 Connell $\mathrm{T}$, Tebruegge M, Ritz N, et al. Indeterminate interferon- $\gamma$ release assay results in children. Pediatr Infect Dis J 2010; 29: 285-286.

4 Landi S, Held HR, Tseng MC. Comparative study of 14C-labeled purified protein derivative from various mycobacteria. Appl Microbiol 1970; 20: 696-709.

5 Lein AD, von Reyn CF, Ravn P, et al. Cellular immune responses to ESAT-6 discriminate between patients with pulmonary disease due to Mycobacterium avium complex and those with pulmonary disease due to Mycobacterium tuberculosis. Clin Diagn Lab Immunol 1999; 6: 606-609.

6 Gey van Pittius NC, Warren RM, van Helden PD. ESAT-6 and CFP10: what is the diagnosis? Infect Immun 2002; 70: 6509-6510.

7 Bamford AR, Crook AM, Clark J, et al. Comparison of Interferongamma release assays and tuberculin skin test in predicting active tuberculosis (TB) in children in the UK - a Paediatric TB Network Study. Arch Dis Child 2010 95: 180-186.

DOI: $10.1183 / 09031936.00025510$

\section{From the authors:}

We do appreciate the comments by $\mathrm{M}$. Tebruegge and coworkers about our recently published manuscript in the European Respiratory Journal [1]. The aim of this reply is to clarify some points in order to interpret better the results of the study, given that we think there were some misunderstandings.

In vitro assays for measuring interferon (IFN)- $\gamma$ released by the T-cells after specific Mycobacterium tuberculosis stimulation have demonstrated promising results in adults and also in children for diagnosing tuberculosis (TB) infection [2, 3]. However, there are discordant results between IFN- $\gamma$ based assays and the tuberculin skin test (TST) that require clarification in order to assess the real utility of the in vitro tests in the management of patients $[2,4]$.

In our study we determined the potential role of nontuberculous mycobacteria (NTM) sensitisation in children as a factor of discordant results between TST and an in vitro T-cell based assay (T.SPOT.TB; Oxford Immunotec, Oxford, UK). We enrolled 21 non-bacille Calmette-Guérin vaccinated paediatric patients for suspicion of latent TB infection (LTBI). These patients yielded a positive TST and a negative T.SPOT.TB. Cells were stimulated with Mycobacterium avium sensitin (MAS) and the presence of reactive T-cells was determined by an ex vivo enzyme-linked immunospot assay. From the 16 patients with a valid result, in 10 cases we obtained a positive ELISPOT result after stimulation with MAS.

Our main disagreement with the argumentation by $\mathrm{M}$. Tebruegge and co-workers resides in the fact that we are not using MAS for distinguishing $M$. tuberculosis from NTM infection. For this objective, we used the specific $M$. tuberculosis RD1 antigens included in the T.SPOT.TB test, and, as no response against RD1 antigens was obtained, we assessed Tcell sensitisation against MAS antigens to investigate if NTM sensitisation could be responsible for TST positivity. Indeed, LEIN et al. [5], also referred to in the letter by M. Tebruegge and co-workers, obtained significant immune responses to ESAT-6 from $59 \%$ of pulmonary $M$. tuberculosis disease patients diagnosed, but no response was obtained from patients with M. avium complex pulmonary disease.

However, we agree with $\mathrm{M}$. Tebruegge and co-workers that in some cases alternative explanation can also be possible. Given that MAS are not totally specific, and cross-reactions with other mycobacteria species have been described, we cannot totally exclude the possibility that we are detecting, in some cases, a response of specific T-cells against some $M$. tuberculosis antigens different from ESAT-6 and CFP-10; or a false-negative result of the T.SPOT.TB.

On the one hand, M. Tebruegge and co-workers have shown some concerns about our group of children with positive TST and positive T.SPOT.TB where $50 \%$ of children responded to the MAS. The results are in concordance with the known crossreaction between MAS and other mycobacteria. Nevertheless, we cannot totally reject simultaneous infection of $M$. tuberculosis and NTM. Furthermore, these results are in total agreement with those obtained by LEIN et al. [5], where they found response against MAS in 24 out of $27 \mathrm{M}$. tuberculosis disease patients.

On the other hand, we want to point out that the main MAS positive results were obtained in children enrolled during LTBI screening at school with TST induration $>5 \mathrm{~mm}$ and $<10 \mathrm{~mm}$. In all these children a complete medical exploration, including clinical and radiographic studies, was performed, and active TB was excluded. In the subsequent contact tracing studies no index case was found. Based on the classical studies performed by NyвоE [6], the main guidelines in this kind of child population consider as a cut-off for $M$. tuberculosis infection a TST induration $\geqslant 10 \mathrm{~mm}$, in order to avoid false-positive TST results induced by NTM immunisation [7]. Nevertheless, indurations $>15 \mathrm{~mm}$ [8] and $20 \mathrm{~mm}$ [9] have been reported in children with NTM infections. Therefore, our results reinforce, in part, the guidelines in that unnecessary chemoprophylaxis treatment in this unexposed population could be avoided, and that IFN- $\gamma$ based assays could help to confirm a positive TST result.

Children from contact-tracing studies truly exposed to an active TB case merit special consideration as they can develop the disease very quickly after primary infection, with the most severe forms prevailing in younger children [10]. For this child population we did not recommend withholding the chemoprophylaxis; 